\title{
NOD1 and NOD2 stimulation triggers innate immune responses of human periodontal ligament cells
}

\author{
DO-IN JEON ${ }^{1}$, SE-RA PARK ${ }^{1}$, MEE-YOUNG AHN $^{1}$, SANG-GUN AHN $^{1}$, \\ JONG-HWAN PARK ${ }^{2}$ and JUNG-HOON YOON ${ }^{1}$ \\ ${ }^{1}$ Department of Pathology and Research Center for Oral Disease Regulation of the Aged, School of Dentistry, \\ Chosun University, Gwangju 501-759; ${ }^{2}$ Department of Biochemistry, College of Medicine, \\ Konyang University, Daejeon 302-718, Republic of Korea
}

Received November 7, 2011; Accepted December 13, 2011

DOI: $10.3892 / \mathrm{ijmm} .2012 .878$

\begin{abstract}
Nod-like receptors (NLRs) are cytosolic sensors for microbial molecules. Nucleotide-binding oligomerization domain (NOD)1 and NOD2 recognize the peptidoglycan derivatives, meso-diaminopimelic acid (meso-DAP) and muramyl dipeptide (MDP), respectively, and trigger host innate immune responses. In the present study, we examined the function of NOD1 and NOD2 on innate immune responses in human periodontal ligament (PDL) cells. The gene expression of NOD1 and NOD2 was examined by RT-PCR. IL-6 and IL- 8 production in culture supernatants was measured by ELISA. Western blot analysis was performed to determine the activation of NF- $\kappa \mathrm{B}$ and MAPK in response to Tri-DAP and MDP. The genes of NOD1 and NOD2 appeared to be expressed in PDL cells. Although the levels of NOD2 expression were weak in intact cells, MDP stimulation increased the gene expression of NOD2 in PDL cells. Tri-DAP and MDP led to the production of IL- 6 and IL- 8 and the activation of NF- $\kappa B$ and MAPK in PDL cells. Toll-like receptor (TLR) stimulation led to increased gene expression of NOD1 and NOD2 in PDL cells. $\mathrm{Pam}_{3} \mathrm{CSK}_{4}$ (a TLR2 agonist) and IFN- $\gamma$ synergized with Tri-DAP and MDP to produce IL- 8 and IL-6 in PDL cells. Our results indicate that NOD1 and NOD2 are functionally expressed in human PDL cells and can trigger innate immune responses.
\end{abstract}

Correspondence to: Dr Jung-Hoon Yoon, Department of Pathology, School of Dentistry, Chosun University, 375 Seosuk-dong, Dong-gu, Gwangju 501-759, Republic of Korea

E-mail: jhyoon@chosun.ac.kr

Dr Jong-Hwan Park, Department of Biochemistry, College of Medicine, Konyang University, 28 Wonang-1-ro, Gwanjeo-dong, Seo-gu, Daejeon 302-718, Republic of Korea

E-mail: jonpark@konyang.ac.kr

Key words: nucleotide-binding oligomerization domain 1, nucleotidebinding oligomerization domain 2, immune response, periodontal ligament cells

\section{Introduction}

Host defense is achieved by two different immune systems, innate and adaptive immunity. Innate immunity is the first line of defense process to protect the host from microbial pathogens and is primarily mediated by phagocytes such as macrophages and dendritic cells (1-4). The innate immunity recognizes microorganisms via a limited number of pattern-recognition receptors (PRRs), which recognize microbial components, known as pathogen-associated molecular patterns (PAMPs). Toll-like receptors (TLRs) and Nod-like receptors (NLRs) are the representative PRRs.

They recognize microbial molecules including bacterial lipoprotein, lipopolysaccharide (LPS), flagellin, and viral nucleic acids, at the cell surface or endosomal membrane and subsequently activate $\mathrm{NF}-\kappa \mathrm{B}$ and MAPK to trigger the inflammatory process (1). In addition to microbial molecules, a variety of endogenous ligands, such as heat shock proteins, high mobility group box 1 (HMGB1), hyaluronan fragments, heparin sulphate, and fibronectin are recognized by TLR 2 or TLR4 (5). In contrast, NLRs are intracellular, cytoplasmic sensors for microbial components and danger signals $(6,7)$. There are 23 NLR family members in humans and at least 34 NLR genes in mice (7). NLRs are expressed in non-immune cells including epithelial and mesothelial cells as well as immune cells. Nucleotide-binding oligomerization domain (NOD)1 and NOD2, the first identified NLRs consist of an $\mathrm{N}$-terminal caspase recruitment domain (CARD), an intermediate NOD, and a C-terminal leucine-rich repeats (LRRs) domain (8). NOD1 and NOD2 recognize the peptidoglycan derivatives, meso-diaminopimelic acid (meso-DAP) and muramyl dipeptide (MDP), respectively (8). After stimulation by their specific bacterial molecules, NOD1 and NOD2 associate with the adaptor molecule, RICK/Rip2/CARDIAK, through CARD-CARD interaction, which leads to activation of NF- $\mathrm{B}$ and MAPK, followed by induction of numerous genes involved in the inflammatory process (9-11).

Periodontitis is a chronic inflammatory disease initiated on the periodontium by toxin and oxygen produced from periodontopathic bacteria (12), which results in tooth loss and periodontal bone resorption because the supportive tissue surrounding the teeth was destructed. Gram-negative bacteria 
such as Porphyromonas gingivalis, Aggregatibacter actinomycetemcomitans and Fusobacterium necleatum have been considered to be important pathogenic microorganisms associated with periodontitis (12-14).

Periodontal ligament (PDL) cells not only function as supporting cells for periodontal tissues but also produce inflammatory mediators that recognize various molecules including LPS (15). There is evidence that TLRs mediate immune responses of PDL cells against periodontal infections $(16,17)$. However, little is known about the role of NOD1 and NOD2 in innate immune responses of PDL cells. Therefore, in the present study, we examined the function of NOD1 and NOD2 in the innate immune responses of human PDL cells.

\section{Materials and methods}

Cell culture and reagents. A human periodontal ligament cell line was a gift from Dr Maeda (Kyushu University Hospital, Fukuoka, Japan). This cell line was immortalized by SV40 T-antigen and hTERT gene transfer (18). THP-1 cells, a human monocytic leukemia cell line, were used as a positive control. PDL cells were cultured in Minimum Essential Medium $\alpha$ (Gibco, Grand Island, NY, USA) containing 1X penicillin/streptomycin and $10 \%$ fetal bovine serum in $5 \% \mathrm{CO}_{2}$ incubator at $37^{\circ} \mathrm{C}$. Tri-DAP, $\mathrm{Pam}_{3} \mathrm{CSK}_{4}$, LPS, and recombinant human IFN- $\gamma$ were purchased from Invivogen, Inc. (San Diego, CA, USA) and muramyl dipeptide [MDP; Ac-(6-O-strearoyl)-muramyl-Ala-DGlu- $\mathrm{NH}_{2}$ ] was from Bachem, Inc. (Torrance, CA, USA).

$R T-P C R$. Total-RNA was extracted from the cell using easyBLUE (Intron Biotechnology, Daejeon, Korea) according to the manufacturer's instruction. One microgram of total-RNA was reverse transcribed into cDNA, and PCR was performed using the Power cDNA Synthesis kit (Intron Biotechnology) and One-step RT-PCR with AccuPower ${ }^{\circledR}$ HotStart PCR PreMix (Bioneer, Daejeon, Korea). The following primer sets were used. Human NOD1, forward, 5'-CCACTTCACAGCTGGAG ACA-3' and reverse, 3'-TGAGTGGAAGCAGCATTTTG-5'; human NOD2, forward, 5'-GAATGTTGGGCACCTCAAGT-3 and reverse, 3'-CAAGGAGCTTAGCCATGGAG-5'; human GAPDH, forward, 5'-GTCGGAGRCAACGGATT-3' and reverse, 3'-AAGCTTCCCGTTCTCAG-3'.

The PCR reaction condition included pre-denaturing at $94^{\circ} \mathrm{C}$ for $30 \mathrm{sec}$, then $35-40$ cycle of $56^{\circ} \mathrm{C}$ for $30 \mathrm{sec}, 72^{\circ} \mathrm{C}$ for $1 \mathrm{~min}$. PCR products were then electrophoresed on a $1.5 \%$ agarose gel and visualized using a gel documentation system.

Measurement of IL-6 and IL-8. The cells in triplicate were treated with the indicated doses of Tri-DAP and MDP or combination with TLR agonist or IFN- $\gamma$ for $24 \mathrm{~h}$ and the culture supernatant was collected. The concentration of IL-6 and IL-8 in the culture supernatants were determined using a commercial ELISA kit (R\&D Systems, Minneapolis, MN, USA)

Western blotting. The cells (1x10\%/well) were plated in $35-\mathrm{mm}$ culture dishes. The cells were treated with $10 \mu \mathrm{g} / \mathrm{ml}$ of Tri-DAP and MDP and were lysed in buffer containing 1\% Nonidet-P40 supplemented with a complete protease inhibitor cocktail (Roche) and $2 \mathrm{mM}$ dithiothreitol. Lysates were resolved by $10 \%$ SDS-PAGE, transferred to a polyvinylidene

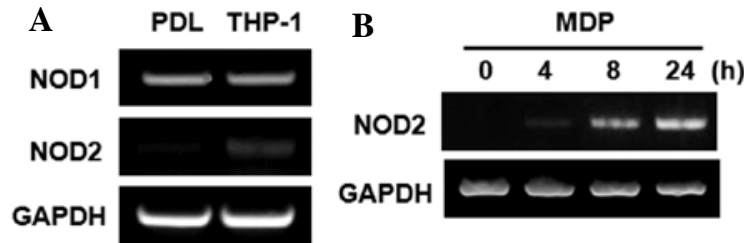

Figure 1. The gene expression levels of NOD1 and NOD2 in PDL cells. (A) The mRNA expression levels of NOD1, NOD2 and GAPDH were examined in human PDL cells by RT-PCR. THP-1 cells were used as a positive control. (B) PDL cells were stimulated with $10 \mu \mathrm{g} / \mathrm{ml}$ of MDP and total-RNA was extracted at the indicated times after stimulation. NOD2 expression levels were determined by RT-PCR.

fluoride (PVDF) membrane, and immunoblotted with primary antibodies against regular- and phospho-IкB- $\alpha$, p38, ERK and JNK (Cell Signaling Technology, Inc., Beverly, MA, USA). After immunoblotting with secondary antibodies, proteins were detected with an enhanced chemiluminescence (ECL) reagent (Intron Biotechnology).

Statistical analysis. The differences among the mean values of the different groups were assessed, and the values are expressed as the mean \pm SD. All of the statistical calculations were performed by one-way ANOVA using the GraphPad Prism version 5.01 (GraphPad Software, San Diego, CA, USA). Values of $\mathrm{P}<0.05$ were considered significant.

\section{Results}

Expression of NOD1 and NOD2 in PDL cells. The gene expression of NOD1 and NOD2 in PDL cells were examined by RT-PCR. THP-1 cells, human monocyte leukemia cells, were used as a positive control. The gene of NOD1 was strongly expressed in PDL cells, and NOD1 levels were comparable to that in THP-1 cells. In contrast, NOD2 expression was found to be low level in PDL cells, as compared to THP-1 cells (Fig. 1A). However, the stimulation with MDP, a specific NOD2 agonist, augmented the gene expression of NOD2 in a time-dependent manner in PDL cells (Fig. 1B).

NOD1 and NOD2 stimulation leads to increased production of IL-6 and IL-8 in PDL cells. To determine whether the stimulation of NOD1 and NOD2 leads to the production of proinflammatory cytokines/chemokines, the cells were treated with Tri-DAP (NOD1 agonist) and MDP (NOD2 agonist) and the production of IL-6 and IL- 8 from culture supernatants was determined by ELISA. Both Tri-DAP and MDP can lead to increased production of IL-6 and IL-8 production in PDL cells in a dose-dependent manner (Fig. 2). Both IL-6 and IL-8 production was more increased by Tri-DAP than MDP, suggesting that NOD1 may play a more important role in the immune response of PDL cells than NOD2.

Tri-DAP and MDP induce NF- $\kappa B$ and MAPK activation in $P D L$ cells. Sensing of microbial molecules by NOD1 and NOD2 induce the activation of NF- $\kappa \mathrm{B}$ and MAPK in various cell types including macrophages (19-21). To determine whether NOD1 and NOD2 stimulation leads to NF- $\kappa$ B and 


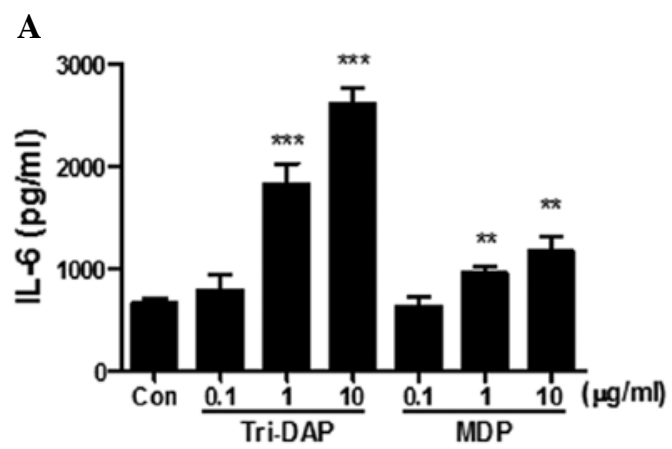

B

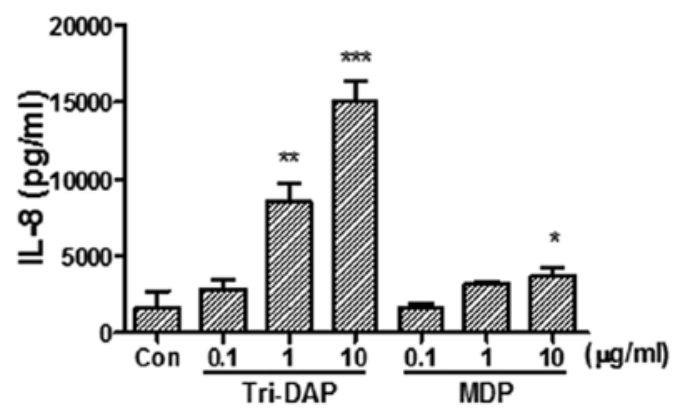

Figure 2. The production of IL-6 and IL- 8 by PDL cells in response to Tri-DAP and MDP. The cells in triplicate were stimulated with the indicated doses of Tri-DAP or MDP for $24 \mathrm{~h}$, and the levels of (A) IL-6 and (B) IL-8 in the culture supernatant were determined using a commercial ELISA kit. ${ }^{*} \mathrm{P}<0.05 ;{ }^{* * *} \mathrm{P}<0.01 ;{ }^{* * * * *} \mathrm{P}<0.001$.

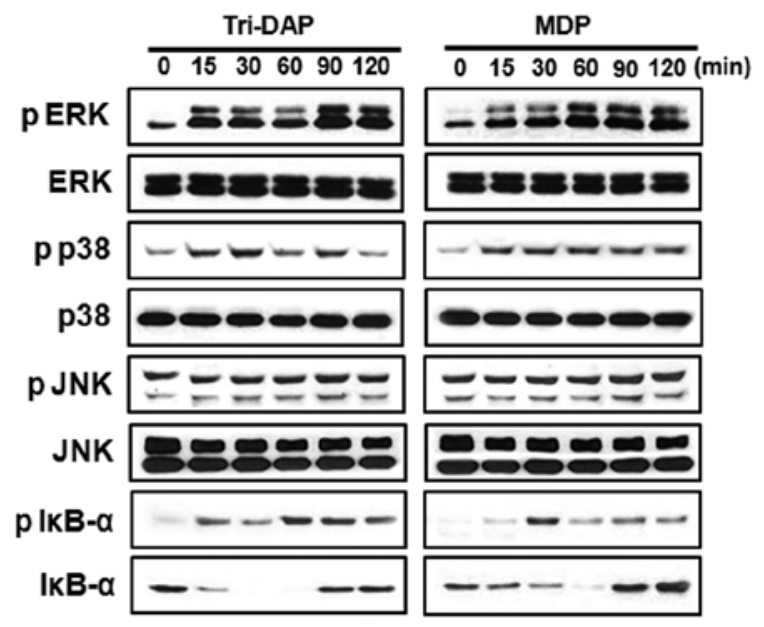

Figure 3. NF- $\mathrm{KB}$ and MAPK activation in PDL cells in response to Tri-DAP and MDP. The cells were stimulated with $10 \mu \mathrm{g} / \mathrm{ml}$ of Tri-DAP or MDP, and protein was extracted at the indicated time points. The total and phosphorylated forms of IкB- $\alpha, \mathrm{p} 38$, JNK and ERK were examined by western blotting.

MAPK activation in PDL cells, the cells were treated with Tri-DAP or MDP and extracts were prepared at different times after stimulation. Subsequently, immunoblotting was performed using antibodies that recognize activated forms of IкB- $\alpha$, p38, JNK and ERK. Results showed that both Tri-DAP and MDP induced phosphorylation of IкB- $\alpha$, p38 and ERK, but not JNK (Fig. 3). The kinetics of IкB- $\alpha$ phosphorylation and degradation were different between Tri-DAP and MDP. Fifteen minutes after stimulation, Tri-DAP induced phosphorylation of IкB- $\alpha$ and maximal phosphorylation was detected at 60 and $90 \mathrm{~min}$ after stimulation. However, MDP induced optimal phosphorylation of $\mathrm{I}-\kappa \mathrm{B} \alpha$ at $30 \mathrm{~min}$ after stimulation, which was reduced after that time (Fig. 3).

TLR stimulation enhances the gene expression of NOD1 and NOD2 and augments the production of IL- 6 and $I L-8$ increased by Tri-DAP and MDP in PDL cells. It has been known that TLRs synergize with NOD1 and NOD2 to produce cytokines in macrophages and dendritic cells $(20,22)$. We first examined whether stimulation by TLRs affects the gene expression of NOD1 and NOD2 in PDL cells. The treatment of LPS (a TLR4 agonist) and $\mathrm{Pam}_{3} \mathrm{CSK}_{4}$ (a TLR2 agonist) could enhance the gene expression of NOD1 and NOD2 beginning at $4 \mathrm{~h}$ after stimulation (Fig. 4A). We next investigated whether
A
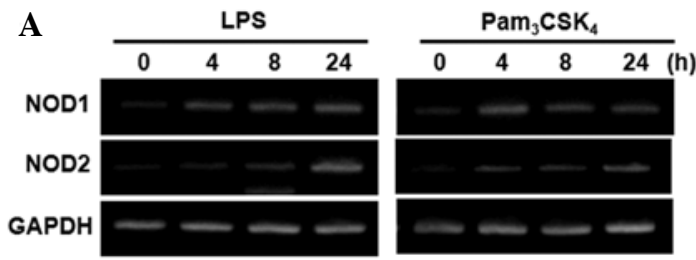

B

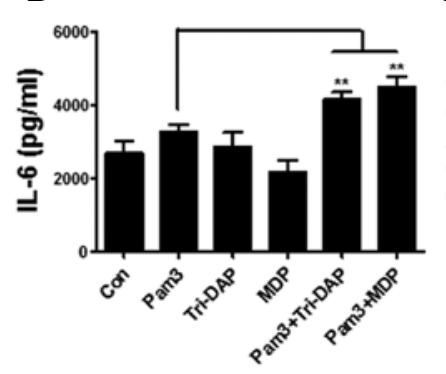

C

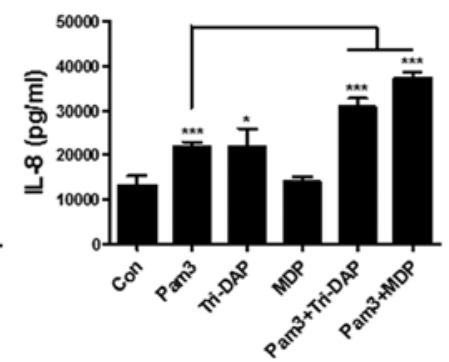

Figure 4. Upregulation of the gene expression of NOD1 and NOD2 by TLR agonists and synergism of NLR and TLR agonists for IL-6 production in PDL cells. PDL cells were stimulated with $1 \mu \mathrm{g} / \mathrm{ml}$ of LPS and $\mathrm{Pam}_{3} \mathrm{CSK}_{4}$ and total-RNA was extracted at the indicated times after stimulation. (A) The gene expression of NOD1 and NOD2 was determined by RT-PCR. PDL cells in triplicate were treated with indicated combination of $\operatorname{Pam}_{3} \mathrm{CSK}_{4}(0.1 \mu \mathrm{g} / \mathrm{ml})$ and Tri-DAP or MDP $(0.1 \mu \mathrm{g} / \mathrm{ml})$ for $24 \mathrm{~h}$ and the levels of (B) IL-6 and (C) IL-8 in the culture supernatant were determined. ${ }^{*} \mathrm{P}<0.05 ;{ }^{* *} \mathrm{P}<0.01 ;{ }^{* * *} \mathrm{P}<0.001$.

activation of TLRs can augment the ability of PDL cells to produce IL- 6 and IL- 8 by Tri-DAP and MDP. Dose response experiments were performed to determine an appropriate dose of $\mathrm{Pam}_{3} \mathrm{CSK}_{4}$ to induce marginal production of IL-6 and IL-8. Results revealed that $0.1 \mu \mathrm{g} / \mathrm{ml}$ of $\mathrm{Pam}_{3} \mathrm{CSK}_{4}$ led to a minor increase of IL-6 and IL-8 production in PDL cells (data not shown), and this concentration was used for further experiments. For the synergism experiment, the cells were treated with indicated agonists alone or their combination for $24 \mathrm{~h}$ and IL-6 and IL-8 production was measured from culture supernatants. As shown in Fig. 4, combination treatment of $\mathrm{Pam}_{3} \mathrm{CSK}_{4}$ and Tri-DAP or MDP significantly augmented IL-6 or IL-8 production in PDL cells, as compared to the single agonisttreated group (Fig. 4B and C).

IFN- $\gamma$ synergizes with Tri-DAP and MDP to produce IL- 6 in $P D L$ cells. Finally, we examined whether IFN- $\gamma$ can augment Tri-DAP and MDP-induced cytokine production by PDL cells. 
A

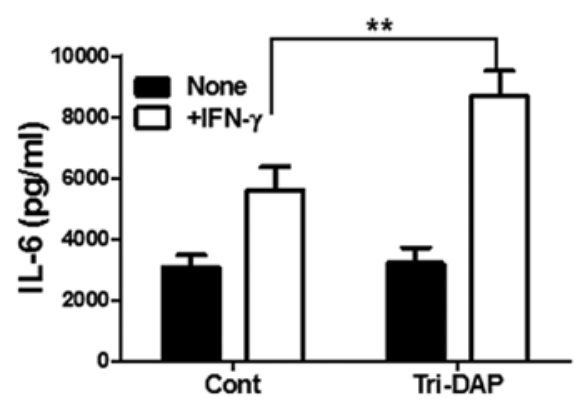

B

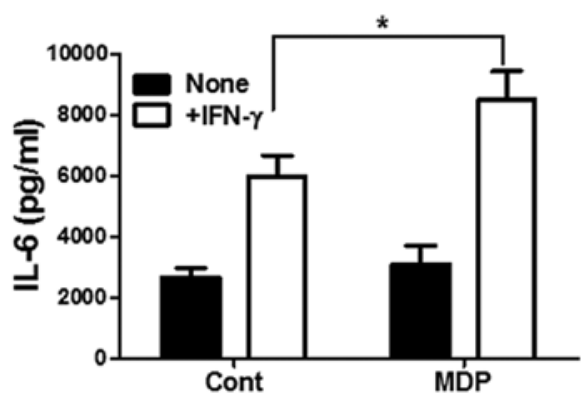

Figure 5. Tri-DAP and MDP augments IFN- $\gamma$-induced IL-6 production in PDL cells. PDL cells in triplicate were treated with IFN- $\gamma(0.1 \mu \mathrm{g} / \mathrm{ml}),(\mathrm{A})$ Tri-DAP $(0.1 \mu \mathrm{g} / \mathrm{ml})$ and $(\mathrm{B}) \mathrm{MDP}(0.1 \mu \mathrm{g} / \mathrm{ml})$ alone or their combination for $24 \mathrm{~h}$ and the levels of IL- 6 in the culture supernatant were determined. ${ }^{*} \mathrm{P}<0.05$; ${ }^{* *} \mathrm{P}<0.01$.

IFN- $\gamma$ alone could increase IL-6 production in PDL cells (Fig. 5A). In addition, co-stimulaion with Tri-DAP upregulated IL-6 production in PDL cells, as compared to IFN- $\gamma$ or Tri-DAP alone (Fig. 5A). Furthermore, combination treatment with MDP and IFN- $\gamma$ also enhanced IL- 6 production by PDL cells, although a low dose of MDP alone $(0.1 \mu \mathrm{g} / \mathrm{ml})$ could not increase IL-6 production (Fig. 5B).

\section{Discussion}

Recent studies have demonstrated that NOD1 and NOD2 are expressed in various cell types that exist within the oral tissues and play a role in triggering immune responses (23-25). In healthy gingival tissues, NOD1 and NOD2 exhibit stronger expression than TLRs (24). NOD1 and NOD2 are also expressed in various oral epithelial cells and the stimulation with iE-DAP and MDP upregulates the gene expression of $\beta$-defensin 2 (24). In human gingival fibroblasts, both NOD1 and NOD2 were strongly expressed and their agonists (FK156 for NOD1, MDP for NOD2) could increase the production of IL-6, IL-8 and MCP-1 via an NF- $\kappa$ B-dependent pathway (25). In addition, Hirao et al (23) showed the gene and protein expression of NOD1 and NOD2 in pulp fibroblasts and that iE-DAP and MDP could produce IL-8, suggesting that NOD1 and NOD2 are functionally expressed in pulp fibroblasts. Tang et al (26) showed the gene and protein expression and localization of NOD1 and NOD2 in human PDL fibroblasts. The activation of NOD1 and NOD2 led to the upregulation of tumor necrosis factor receptor-associated factor 6 (TRAF6) and pro-inflammatory cytokines in human PDL cells (26).

In the present study, we revealed that NOD1 is strongly expressed in PDL cells and the expression level is comparable to that in THP-1 cells. NOD2 expression was relatively weak in PDL cells. The expression level of NOD2 varies between cell types. The mRNA and protein of NOD2 was markedly expressed in hepatocytes, oral epithelial cells, and renal tubular epithelial cells (27-29), but was not expressed or was weakly expressed in intestinal epithelial cells (30). In addition, NOD2 expression seems to be regulated by specific treatment. Bacterial flagellin (a TLR5 agonist), E. coli, and IL-1 $\beta$ increased the gene expression of NOD2 in intestinal epithelial cells (30). Furthermore, in the presence of histamine, MALP-2 (a TLR2 agonist), peptidoglycan, and $\beta$-glucan also enhanced NOD2 gene expression in keratinocytes (31). In this study, the gene expression of NOD2 was upregulated by MDP stimula- tion, suggesting that NOD2 may be inducible in PDL cells. In addition, the activation of NOD1 and NOD2 with Tri-DAP and MDP led to IL-6 and IL-8 production and the activation of NF- $\kappa \mathrm{B}$ and MAPK in PDL cells, indicating that NOD1 and NOD2 may be functionally expressed in PDL cells.

Previous studies showed that NOD1 and NOD2 have the synergistic or additive effect with TLRs to produce cytokines and chemokines in immune cells and mesothelial cells $(20,21,32)$. These phenomena were also found in several epithelial cells. In oral epithelial cells, NOD1 and NOD2 agonists in combination with TLR agonists synergistically enhanced $\beta$-defensin 2 secretion (33). Moreover, LPS pretreatment enhanced the activation of NF- $\kappa \mathrm{B}$, ERK and JNK by MDP in hepatocytes (27). Likewise, in this study, NOD1 and NOD2 agonists (Tri-DAP and MDP) synergized with a TLR2 agonist $\mathrm{Pam}_{3} \mathrm{CSK}_{4}$ to produce IL-6 and IL-8 in PDL cells. Our results indicate that NOD1 and NOD2 may cooperate with TLRs to elicit immune responses in PDL cells.

IFN $-\gamma$ is known to increase the expression of NOD1 and NOD2 in macrophages $(34,35)$. In addition, IFN- $\gamma$ is essential for a NOD1 agonist, KF1B-induced nitric oxide production in mesothelial cells (21). Therefore, we examined whether IFN- $\gamma$ augments IL-6 production by NOD1 and NOD2 activation in PDL cells. Results showed that the co-stimulation with IFN- $\gamma$ and Tri-DAP or MDP upregulated IL-6 production in PDL cells, as compared to IFN- $\gamma$ or the agonist alone-treated group. These findings suggest that IFN- $\gamma$ may enhance the innate immune response mediated by NOD1 and NOD2 signaling in PDL cells.

In conclusion, we reported here that NOD1 and NOD2 are functionally expressed in human PDL cells and the activation of these receptors can induce innate immune responses such as cytokine production and the activation of NF- $\mathrm{NB}$ and MAPK. In addition, our results revealed that TLRs can synergize with NOD1 and NOD2 to produce proinflammatory cytokines/ chemokines. Similarly to immune responses, NOD1 and NOD2 signaling can mediate cellular physiological functions, such as proliferation and differentiation (36-38). Future studies should clarify the function of NOD1 and NOD2 on the cellular physiology of PDL cells.

\section{Acknowledgements}

This study was supported by a grant by the Korea Science and Engineering Foundation (KOSEF) funded by the Korean government (MOST) (grant no. R13-2008-010-00000-0). 


\section{References}

1. Akira S, Uematsu S and Takeuchi O: Pathogen recognition and innate immunity. Cell 124: 783-801, 2006.

2. Hoffmann JA: The immune response of Drosophila. Nature 426: 33-38, 2003.

3. Beutler B, Eidenschenk C, Crozat K, Imler JL, Takeuchi O, Hoffmann JA and Akira S: Genetic analysis of resistance to viral infection. Nat Rev Immunol 7: 753-766, 2007.

4. Medzhitov R: Recognition of microorganisms and activation of the immune response. Nature 449: 819-826, 2007.

5. Yu L, Wang L and Chen S: Endogenous toll-like receptor ligands and their biological significance. J Cell Mol Med 14: 2592-2603 2010.

6. Chen G, Shaw MH, Kim YG and Nunez G: NOD-like receptors: role in innate immunity and inflammatory disease. Annu Rev Pathol 4: 365-398, 2009.

7. Franchi L, Warner N, Viani K and Nunez G: Function of Nod-like receptors in microbial recognition and host defense. Immunol Rev 227: 106-128, 2009.

8. Inohara N, Chamaillard M, McDonald C and Nunez G: NOD-LRR proteins: role in host-microbial interactions and inflammatory disease. Annu Rev Biochem 74: 355-383, 2005.

9. Inohara N, Koseki T, Lin J, del Peso L, Lucas PC, Chen FF, Ogura Y and Núñez G: An induced proximity model for NF- $\mathrm{B}$ activation in the Nod1/RICK and RIP signaling pathways. J Biol Chem 275: 27823-27831, 2000.

10. Girardin SE, Tournebize R, Mavris M, Page AL, Li X, Stark GR, Bertin J, DiStefano PS, Yaniv M, Sansonetti PJ and Philpott DJ: CARD4/Nod1 mediates NF- $\mathrm{B}$ and JNK activation by invasive Shigella flexneri. EMBO Rep 2: 736-742, 2001.

11. Hayden MS and Ghosh S: Signaling to NF-кB. Genes Dev 18 2195-2224, 2004.

12. Nishihara T and Koseki T: Microbial etiology of periodontitis. Periodontol 2000 36: 14-26, 2004.

13. Slots J, Reynolds HS and Genco RJ: Actinobacillus actinomycetemcomitans in human periodontal disease: a cross-sectional microbiological investigation. Infect Immun 29: 1013-1020, 1980

14. Socransky S, Haffajee A, Cugini M, Smith C and Kent R Jr: Microbial complexes in subgingival plaque. J Clin Periodontol 25: 134-144, 1998

15. Yamaji Y, Kubota T, Sasaguri K, Sato S, Suzuki Y, Kumada H and Umemoto T: Inflammatory cytokine gene expression in human periodontal ligament fibroblasts stimulated with bacterial lipopolysaccharides. Infect Immun 63: 3576-3581, 1995.

16. Sun Y, Shu R, Li CL and Zhang MZ: Gram-negative periodontal bacteria induce the activation of Toll-like receptors 2 and 4 , and cytokine production in human periodontal ligament cells. J Periodontol 81: 1488-1496, 2010.

17. Sun Y, Shu R, Zhang MZ and Wu AP: Toll-like receptor 4 signaling plays a role in triggering periodontal infection. FEMS Immunol Med Microbiol 52: 362-369, 2008.

18. Fujii S, Maeda H, Wada N, Kano Y and Akamine A: Establishing and characterizing human periodontal ligament fibroblasts immortalized by SV40T-antigen and hTERT gene transfer. Cell Tissue Res 324: 117-125, 2006.

19. Kobayashi KS, Chamaillard M, Ogura Y, Henegariu O, Inohara N, Nuñez G and Flavell RA: Nod2-dependent regulation of innate and adaptive immunity in the intestinal tract. Science 307: 731-734, 2005

20. Park JH, Kim YG, McDonald C, Kanneganti TD, Hasegawa M, Body-Malapel M, Inohara N and Núñez G: RICK/RIP2 mediates innate immune responses induced through Nod1 and Nod2 but not TLRs. J Immunol 178: 2380-2386, 2007.

21. Park JH, Kim YG, Shaw M, Kanneganti TD, Fujimoto Y, Fukase K, Inohara N and Núñez G: Nod1/RICK and TLR signaling regulate chemokine and antimicrobial innate immune responses in mesothelial cells. J Immunol 179: 514-521, 2007.
22. Tada H, Aiba S, Shibata KI, Ohteki T and Takada H: Synergistic effect of Nod1 and Nod2 agonists with toll-like receptor agonists on human dendritic cells to generate interleukin-12 and T helper type 1 cells. Infect Immun 73: 7967-7976, 2005.

23. Hirao K, Yumoto H, Takahashi K, Mukai K, Nakanishi T and Matsuo T: Roles of TLR2, TLR4, NOD2, and NOD1 in pulp fibroblasts. J Dent Res 88: 762-767, 2009.

24. Sugawara Y, Uehara A, Fujimoto Y, Kusumoto S, Fukase K, Shibata K, Sugawara S, Sasano T and Takada H: Toll-like receptors, NOD1, and NOD2 in oral epithelial cells. J Dent Res 85: 524-529, 2006.

25. Uehara A and Takada H: Functional TLRs and NODs in human gingival fibroblasts. J Dent Res 86: 249-254, 2007.

26. Tang L, Zhou XD, Wang Q, Wang Y, Li XY and Huang DM: Expression of TRAF6 and pro-inflammatory cytokines through activation of TLR2, TLR4, NOD1, and NOD2 in human periodontal ligament fibroblasts. Arch Oral Biol 56: 1064-1072, 2011.

27. Scott MJ, Chen C, Sun Q and Billiar TR: Hepatocytes express functional NOD1 and NOD2 receptors: a role for NOD1 in hepatocyte CC and CXC chemokine production. J Hepatol 53: 693-701, 2010.

28. Shigeoka AA, Kambo A, Mathison JC, King AJ, Hall WF, da Silva Correia J, Ulevitch RJ and McKay DB: Nod1 and nod2 are expressed in human and murine renal tubular epithelial cells and participate in renal ischemia reperfusion injury. J Immunol 184: 2297-2304, 2010.

29. Sugawara Y, Uehara A, Fujimoto Y, Kusumoto S, Fukase K, Shibata K, Sugawara S, Sasano T and Takada H: Toll-like receptors, NOD1, and NOD2 in oral epithelial cells. J Dent Res 85: 524-529, 2006.

30. Begue B, Dumant C, Bambou JC, Beaulieu JF, Chamaillard M, Hugot JP, Goulet O, Schmitz J, Philpott DJ, Cerf-Bensussan N, et al: Microbial induction of CARD15 expression in intestinal epithelial cells via toll-like receptor 5 triggers an antibacterial response loop. J Cell Physiol 209: 241-252, 2006.

31. Kobayashi M, Yoshiki R, Sakabe J, Kabashima K, Nakamura M and Tokura Y: Expression of Toll-like receptor 2, NOD2 and dectin-1 and stimulatory effects of their ligands and histamine in normal human keratinocytes. Br J Dermatol 160: 297-304, 2009.

32. van Heel DA, Ghosh S, Butler M, Hunt K, Foxwell BM, MenginLecreulx D and Playford RJ: Synergistic enhancement of Toll-like receptor responses by NOD1 activation. Eur J Immunol 35: 2471-2476, 2005

33. Uehara A and Takada H: Synergism between TLRs and NOD1/2 in oral epithelial cells. J Dent Res 87: 682-686, 2008.

34. Totemeyer S, Sheppard M, Lloyd A, Roper D, Dowson C, Underhill D, Murray P, Maskell D and Bryant C: IFN- $\gamma$ enhances production of nitric oxide from macrophages via a mechanism that depends on nucleotide oligomerization domain-2. J Immunol 176: 4804-4810, 2006.

35. Hisamatsu T, Suzuki M and Podolsky DK: Interferon- $\gamma$ augments CARD4/NOD1 gene and protein expression through interferon regulatory factor-1 in intestinal epithelial cells. J Biol Chem 278: 32962-32968, 2003.

36. Kim HS, Shin TH, Yang SR, Seo MS, Kim DJ, Kang SK, Park JH and Kang KS: Implication of NOD1 and NOD2 for the differentiation of multipotent mesenchymal stem cells derived from human umbilical cord blood. PLoS One 5: e15369, 2010.

37. Petterson T, Jendholm J, Mansson A, Bjartell A, Riesbeck K and Cardell LO: Effects of NOD-like receptors in human B lymphocytes and crosstalk between NOD1/NOD2 and Toll-like receptors. J Leukoc Biol 89: 177-187, 2011.

38. Cruickshank SM, Wakenshaw L, Cardone J, Howdle PD, Murray PJ and Carding SR: Evidence for the involvement of NOD2 in regulating colonic epithelial cell growth and survival. World J Gastroenterol 14: 5834-5841, 2008. 